Short communication

\title{
A comprehensive molecular approach to the detection of drug-type versus fiber-type hemp varieties
}

\author{
Giuseppe Forlani $^{\mathrm{a}, *}$, Davide Petrollino ${ }^{\mathrm{b}}$ \\ ${ }^{a}$ Department of Life Science and Biotechnology, University of Ferrara, via L. Borsari 46, Ferrara, IT, 44121, Italy \\ ${ }^{\mathrm{b}}$ Vitelium GmbH, Stresemannstrasse 305, Hamburg, D 22761, Germany
}

\section{A R T I C L E I N F O}

\section{Keywords:}

Cannabis sativa L.

Fiber hemp

Drug hemp

Chemotype

Single nucleotide polymorphism

Molecular discrimination

Polymerase chain reaction

\begin{abstract}
A B S T R A C T
The availability of molecular markers able to distinguish drug-type from fiber-type Cannabis sativa cultivars would allow fast and cheap analysis of any plant specimen, including seeds and leaves. Several approaches to this issue have been described, mainly using polymorphisms in the genes coding for tetrahydrocannabinol acid synthase or cannabidiolic acid synthase. Some studies reported sequencing of these genes from small groups of hemp varieties belonging to both chemotypes, showing the occurrence of specific DNA signatures. However, the effectiveness of the corresponding primers to discriminate among chemotypes has been validated on a limited number of cultivars, or not tested at all. Here we report a thorough in silico analysis of available gene sequences for both synthases, showing the existence of hypervariable regions at 3' and 5' ends. This notwithstanding, some possible signatures were identified, and 12 putatively specific primer pairs were designed and tested on 16 fibertype and 11 drug-type varieties. In most cases inconsistent results were obtained, further strengthening the high genetic variability of these genes in hemp germplasm, yet some highly informative polymorphisms were identified. Potentiality and perspectives of this approach are discussed.
\end{abstract}

\section{Introduction}

Hemp (Cannabis sativa L.) is attracting increasing interest as a sustainable industrial crop for fibers to replace cotton or synthetic materials in a variety of applications [1]. In the last century hemp cultivation had been substantially discontinued worldwide, mainly because of banning laws that did not differentiate industrial hemp from marijuana (Cannabis indica L.) [2]. In recent years many countries reintroduced hemp and legalized its production as an agricultural commodity, leading to a renaissance of this crop [3]. However, some problems limit the sector revival [4], among which the unavailability of easy, fast and cheap analytical methods to distinguish between (legal) fiber and (in most cases still prohibited [5]) drug varieties. The two chemotypes differ for their content of $\delta^{9}$-tetrahydrocannabinol (THC), one of a hundred cannabinoids identified in Cannabis spp. Fiber-type cultivars should contain low amounts of this addictive compound, and higher concentration of cannabidiol (CBD). Although some simple immunological methods for THC detection have been proposed [6], reliable cannabinoids quantitation requires complex protocols of extraction and analysis [e.g. 7]. Moreover, the levels of these substances differ significantly among plant tissues [8], being synthesized and accumulated mainly in floral organs [9], a fact that hampers the possibility of analysing other specimens, such as leaves and seeds.

In this view, the availability of molecular markers discriminating drug-type from fiber-type hemp varieties would be of great interest. Several studies have been undertaken with this aim, using for instance inter simple sequence repeats analysis [10] or the RAPD method [11]. Since single nucleotide polymorphism (SNP) assay would allow simpler and faster discrimination, in their pioneer work [12] Kojoma and co-workers studied the occurrence of SNPs in the genomic DNA sequence for $\delta^{9}$-tetrahydrocannabinolic acid synthase (THCAS), the enzyme channeling the intermediate cannabigerolic acid toward THC synthesis [13]. By sequencing THCAS from 6 drug-type and 7 fiber-type varieties, a specific PCR marker for the drug-type strains was identified [12]. A similar approach was used on another small group of 12 drug-type and 4 fiber-type Moroccan isolates [14]. Four SNPs within a $399 \mathrm{bp}$ fragment among those described by Kojoma and co-workers were used to distinguish putatively active and inactive THCAS. It was claimed that this assay was able to differentiate chemotypes when used to screen a hundred hemp varieties [15], but no confirmative data were reported.

\footnotetext{
* Corresponding author.

E-mail address: flg@unife.it (G. Forlani).
} 
Table 1

Putatively specific primers to amplify hemp THCAS and CBDAS sequences. The expected formation of amplicons is indicated.

\begin{tabular}{|c|c|c|c|c|c|c|c|}
\hline \multirow{2}{*}{ Pair } & \multirow{2}{*}{ Primer, forward } & \multirow{2}{*}{ Primer, reverse } & \multirow{2}{*}{ size } & \multicolumn{2}{|l|}{ CBDAS } & \multicolumn{2}{|l|}{ THCAS } \\
\hline & & & & fiber cluster & drug cluster & fiber cluster & drug cluster \\
\hline A & GAATCTGTATTTGTCCAAA & AAGGAGTCATGAAGTTAT & 235 & $\square$ & & & \\
\hline B & CACTATTCTATGCTCCAAGAAA & GTAGACTTTGGGACAGCA & 480 & $\nabla$ & & & \\
\hline C & AGAATCTGTATTTGTCCAAA & TTCCTATATCAAGGTCTCTA & 294 & $\square$ & & & \\
\hline $\mathrm{D}$ & CACTATTCTATGTCCAAGAAAA & AAGTGTGCATCAACGATATT & 341 & & $\square$ & & \\
\hline $\mathrm{E}$ & CCAATGTAACAAATCTAAA & TTGAATGCATGTTTCTCA & 277 & & $\square$ & & \\
\hline $\mathrm{F}$ & CAAGGCACTATTCTATGTC & AGACTTTGTTGGGACAGC & 485 & & $\square$ & & \\
\hline G & GCTATAGTAGACTTGAGAAA & TGAGTCGTGAGCATTAAA & 494 & & & $\square$ & \\
\hline $\mathrm{H}$ & TCAAAGTAGATATTCATAGCCAAA & AAGTGAGTCGTGAGCATTAAA & 466 & & & $\square$ & \\
\hline I & TAAGAAACTAATACCTGAAA & ACATAAGGAGTTGTGAAA & 255 & & & $\nabla$ & \\
\hline $\mathrm{J}$ & TACATGGTTACTTCTCTTCAA & AATTGGTTTCTTAACATAGTCTAA & 251 & & & & $\square$ \\
\hline $\mathrm{K}$ & ACTCACTTCATAACAAAGAA & ACTTAATTGAGAAAGCCGT & 283 & & & & $\square$ \\
\hline $\mathrm{L}$ & TATTATTGATGCACACTTAGT & AAAATTTACAACACCACTG & 472 & & & & $\square$ \\
\hline $\mathrm{T}$ & TTGGAGAAGTTTATTATTGG & ACTAGACTATCCACTCCACCA & 510 & $\nabla$ & $\square$ & $\square$ & $\square$ \\
\hline
\end{tabular}

Moreover, THC content in hemp floral tissues does not seem to depend on the presence of active $v s$ inactive THCAS forms, but on the competition for cannabigerolic acid among THCAS and other two enzymes catalyzing its oxidocyclization, cannabidiolic acid synthase (CBDAS) and cannabichromenic acid synthase [16]. CBDAS, which directs the precursor into the branch of the biosynthetic pathway leading to CBD, shows a surprising level of homology to THCAS, with about 80-85\% identity in a 550-amino acid overlap [17]. Also based on the results of a genetic analysis showing an approximately 1:2:1 segregation of chemotypes [18], it was therefore hypothesized that THCAS and CBDAS were allelic and co-dominant [17]. When this co-dominant marker system was applied to a larger number of samples in commercially available material, it apparently discriminated most of 25 fiber-type from 12 drug-type cultivars [19]. The analysis of 10 drug-type and 8 fiber-type accessions found out that in several cases more than one expressed sequence for THCAS and CBDAS was present, although the respective transcription rate did not correlate with the resulting proportion of THCA and CBDA. Some SNPs were identified in both alleles, which were found to relate to the cannabinoid composition of the inflorescence, and were thus proposed to have a functional significance [20]. The evaluation of an F2 population resulting from the cross of marijuana and hemp varieties pointed out the presence in the former of two CBDAS nonfunctional homologs, leading to conclude that plants that are homozygous for functional CBDAS lack the capacity to accumulate THC [21]. This notwithstanding, neither the general occurrence of $C B D A S$ containing premature stop codons or frame shift mutations in drug-type cultivars, nor the possible existence of distinctive DNA signatures to be used for SNPs analysis were investigated.

All these studies were in part superseded by recent sequencing data providing conclusive evidence that the THCAS and CBDAS scaffolds are not allelic, but at separate loci, though adjacent on the same chromosome [16]. On this basis, CBDAS and THCAS from 11 drug-type and 10 fiber-type hemp varieties were sequenced, allowing the identification of multiple genetic markers that discriminated between the two chemotypes. In particular, four functional SNPs that were hypothesized to induce decreased THCAS activity in the fiber-type plants, and a deletion in the CBDAS gene possibly resulting in loss of function of the enzyme in the drug-type varieties were reported [22]. This work was remarkable in that the identification of markers was based on both THCAS and CBDAS, yet neither an in silico analysis on other available sequences, nor a confirmative PCR assay was performed. Moreover, a large scale single molecule sequencing of THCAS showed the occurrence of a highest genetic heterogeneity in drug varieties, both for gene copy number and sequence variation [23], questioning the possibility of chemotype prediction based on simple molecular markers.

To address this point, we analysed all CBDAS and THCAS sequences described in previous papers [12-14,17,19-23] and available in public databases. Some putatively discriminating primers were designed, and verified on a group of 27 mostly unsequenced, commercial hemp varieties of both chemotypes.

\section{Materials and methods}

\subsection{In silico analysis}

Sequences for C. sativa THCAS and CBDAS that had been described in previous studies [12-14,17,19-23] were retrieved from GenBank (https://www.ncbi.nlm.nih.gov/genbank/), and other sequences available in databanks were found using BLAST (https://blast.ncbi.nlm.nih. gov/ Blast.cgi) analysis [24], with default settings. The accession numbers of the 145 THCAS and 38 CBDAS genes considered are reported in Supplementary Table S1. Sequences were aligned using Clustal Omega (https://www.ebi.ac.uk/Tools/msa/clustalo/) [24]. The resulting Neighbour-joining tree was visualized with T-REX [25].

\subsection{Primer design}

Primers were designed with the PRISE2 software [26] with the following settings: primer length 18-24 bp, amplicon size 200-500 bp, melting temperature $48-58{ }^{\circ} \mathrm{C}$, GC content $40-60 \%$.

\subsection{Plant samples}

Seeds of fiber-type hemp varieties (\#01-16) were purchased on the European market. Seeds of drug-type varieties (\#17-27) were obtained from Sensi Seeds (Oudezijds Achterburgwal 131, 1012DE Amsterdam, The Netherlands).

\subsection{Isolation of DNA}

DNA was isolated from a single seed using the REDExtract-N-Amp ${ }^{\mathrm{TM}}$ Plant PCR Kit (Sigma XNAP). The seed coat was crushed with tweezers in a $0.2-\mathrm{mL}$ thin wall PCR tube, and the material was resuspended in $0.1 \mathrm{~mL}$ of extraction solution. The solubilization was allowed to proceed for $10 \mathrm{~min}$ at $95{ }^{\circ} \mathrm{C}$, then samples were brought back to room temperature and immediately diluted with $0.1 \mathrm{~mL}$ of neutralizing solution. DNA concentration was not quantified, and extracts were stored at $4 \pm 1{ }^{\circ} \mathrm{C}$. Just before the analysis, samples were centrifuged $30 \mathrm{~s}$ at 12,000g. Alternatively, DNA was extracted and purified using the E.Z.N. A. Plant DNA Kit (Omega Bio-tek), according to the manifacturer's instructions. In this case, DNA was quantified with a BioSpec-nano Microvolume UV-vis Spectrophotometer (Shimadzu Biotech). In both cases, 3 biological replications were carried out. 


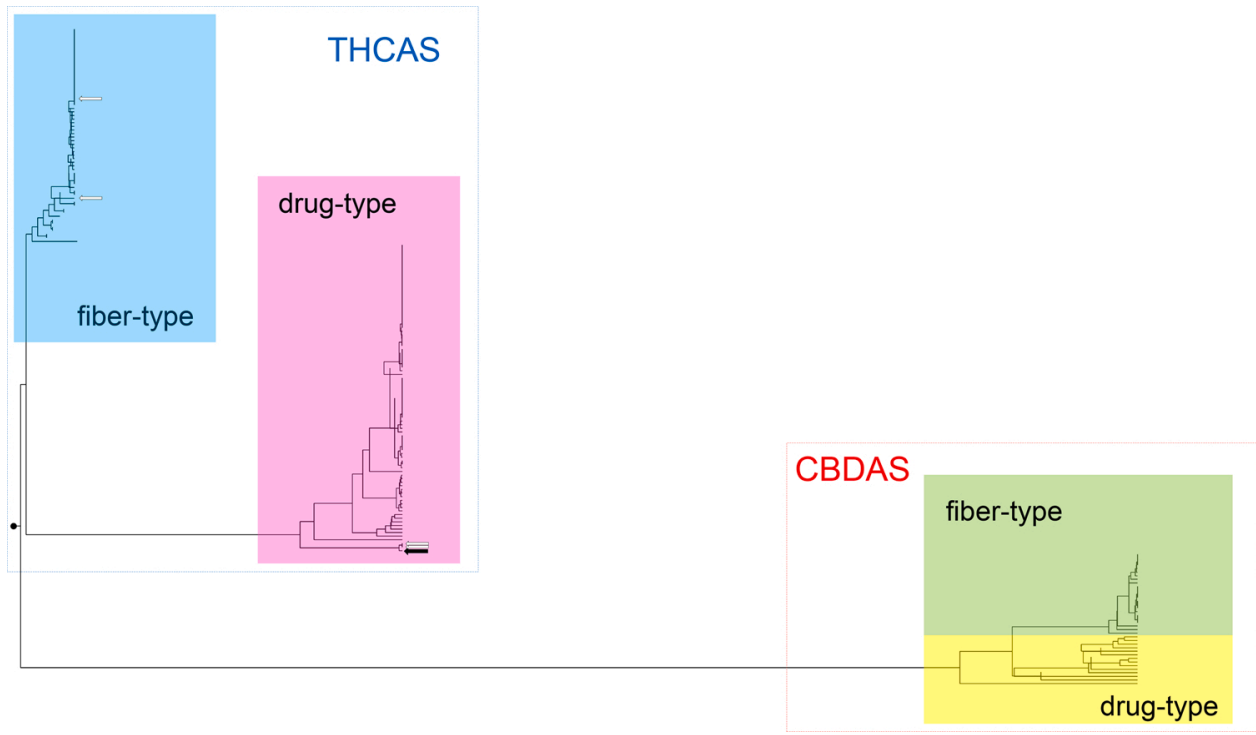

Fig. 1. Neighbour-joining tree generated from the aligned sequences of all hemp THCAS and $C B D A S$ available from public databases. The black arrow points at the only sequence (accession number AB292683.1) clustering with THCAS but annotated as "CBDAS homologue". White arrows show the fiber-type and the drug-type accessions that cluster in the opposite clade. A more analytical picture with all accession numbers and the position of the sequences cloned from plants of known chemotype is provided as Supplementary Fig. S2.

\section{5. $P C R$}

PCRs were carried out in a final volume of $15 \mu \mathrm{L}$ containing $7.5 \mu \mathrm{L}$ of 2X REDExtract-N-Amp ${ }^{\mathrm{TM}}$ PCR ReadyMix ${ }^{\mathrm{TM}}$ (Sigma R4775) or JumpStart ${ }^{\mathrm{TM}}$ REDTaq ${ }^{\circledR}$ ReadyMix $^{\mathrm{TM}}$ Reaction Mix (Sigma P0982), $0.5 \mu \mathrm{M}$ each of forward and reverse primers (Table 1 ) and $3 \mu \mathrm{L}$ of template DNA. Unless specified otherwise, PCR conditions were $95^{\circ} \mathrm{C}$ for 3 min and 35 cycles of $94{ }^{\circ} \mathrm{C}$ for $1 \mathrm{~min}, 49^{\circ} \mathrm{C}$ for $30 \mathrm{~s}$, and $72{ }^{\circ} \mathrm{C}$ for $1 \mathrm{~min}$, with a final extension at $72{ }^{\circ} \mathrm{C}$ for $5 \mathrm{~min}$. Samples were then brought to $4{ }^{\circ} \mathrm{C}$ and immediately analysed.

\subsection{Analysis of PCR products}

Amplification products were separated by electrophoresis on $1 \%$ agarose gels run at $44 \mathrm{~V}$, stained with a 1:5000 dilution of the fluorescent dye Nancy-520 (Sigma 01494), and visualized under blue light. Images were acquired with a Gel Doc 2000 system and the Quantity One software (Bio-Rad). Semi-quantitative evaluation of amplification products was obtained by measurement of band intensities using the ImageJ software [27].

\section{Results}

\subsection{In silico analysis of THCAS and CBDAS sequences from fiber-type and drug-type hemp varieties}

A total of 38 CBDAS and 145 THCAS C. sativa sequences were retrieved from public databases. For 36 and 67 of them information about the chemotype of the plant from which the gene had been sequenced was also available in the literature, respectively (Supplementary Table S1). The sequences were aligned (Supplementary Fig. S1), showing a relatively large, highly conserved central region between approximately residues 400 and 750 , with $69 \%$ identity over a 346 bp stretch. On the contrary, at both 5' and 3' ends no identities were found. A Neighbour-joining tree generated from the aligned sequences (Fig. 1) clearly disclosed two distinct clusters, as expected. A first, more homogeneous group contained all CBDAS but one, the second one included all THCAS as well as accession number AB292683.1, which had been annotated as a "CBDAS homologue". Interestingly, within each cluster two subgroups were evident. In the case of $C B D A S$, although at a low phylogenetic distance, the two subsections perfectly resolved all genes of drug-type varieties from those of fiber-type cultivars. For THCAS, two clades at a more remarkable genetic distance were found.
Also in this case most sequences of a given chemotype clustered together: in the first clade 23 genes belonging to fiber-type cultivars were present, along with the drug-type accessions KJ469379.1 and JQ437490.1, whereas in the second clade 40 genes belonging to the drug-type cultivars were present, plus the fiber-type accessions KJ469381.1 and KJ469383.1 (Supplementary Fig. S2). Results suggest that, following the duplication of an ancestral cannabinoid acid synthase that uses cannabigerolic acid as a substrate, the two paralogs evolved separately leading to a different enzymatic product from the same precursor, i.e. to THCA or CBDA. In both paralogs a divergent evolution has thereafter occurred that most likely caused a prevalent activity of either enzyme, leading to the alternative accumulation of THC or CBD.

\subsection{Design of putatively specific primers for either gene and chemotype}

The obtained picture was consistent with the possibility of finding genetic signatures that would allow chemotype discrimination. To identify specific primers, at first an overall analysis with all CBDAS and THCAS sequences was carried out. Both genes were considered in order to rule out the possibility that a primer couple able to distinguish fibertype from drug-type CBDAS would cause amplification of a corresponding sequence in THCAS, and vice-versa. In other terms, a clade was used as target cluster, and the other clade of the same gene and both clades of the other gene were defined as non-target sequences, in all four possible combinations. However, because of the high genetic variability and the large number of sequences, no discriminating primers were recognized in such a way using the PRISE2 software. To overcome this difficulty, the analysis was repeated using only 8 sequences, two for each group: fiber-type (MG996405.1 and AB212830.1) and drug-type (KJ469378.1 and JQ437491.1) THCAS, and fiber-type (MG996434.1 and KP970859.1) and drug-type (KJ469376.1 and KJ469375.1) CBDAS, respectively. With this approach, 12 putatively specific primer pairs were identified (Table 1). The presence of primer target sequences in most -if not all-genes of the same cluster was then verified, as well as the occurrence of at least 2-3 mismatches in the sequences of the other 3 clusters (Supplementary Fig. S3A-L). The position of each primer pair within the two aligned genes is shown in Supplementary Fig. S4.

\subsection{Validation of the identified SNPs as chemotype markers}

The presence of a conserved sequence in all genes of a cluster, coupled with the presence of some mismatches in the genes of the other clusters, should allow molecular discrimination. However, available 
Table 2

Amplification patterns obtained with the putatively specific primers using DNA template from various fiber-type and drug-type hemp varieties.

\begin{tabular}{|c|c|c|c|c|c|c|c|c|c|c|c|c|c|c|}
\hline$\#$ & Cultivar Primer pair & A & B & C & $\mathrm{D}$ & $\mathrm{E}$ & $\mathrm{F}$ & G & $\mathrm{H}$ & I & $\mathrm{J}$ & $\mathrm{K}$ & $\mathrm{L}$ & $\mathrm{T}$ \\
\hline \multicolumn{15}{|c|}{ fiber-type } \\
\hline 01 & Carmagnola selezionata & +++ & ++++ & ++ & ++++ & - & ++++ & ++++ & ++++ & + & + & ++ & ++++ & +++ \\
\hline 02 & Fedora 17 & + & ++++ & + & - & - & - & - & ++++ & - & - & - & - & - \\
\hline 03 & Felina 32 & +++ & ++++ & ++ & - & - & - & ++ & ++++ & + & - & - & +++ & +++ \\
\hline 04 & Fibrol & ++++ & ++++ & +++ & +++ & ++ & +++ & +++ & ++++ & ++ & + & - & +++ & +++ \\
\hline 05 & Futura 75 & +++ & ++++ & ++ & - & - & - & ++ & ++++ & - & - & - & ++ & +++ \\
\hline 06 & Jubileu Secuieni & + & +++ & - & - & - & - & - & ++++ & - & - & - & + & ++ \\
\hline 07 & KC Dora & ++++ & ++++ & ++ & - & - & - & ++ & +++ & + & - & - & +++ & +++ \\
\hline 08 & KC Zuzana C1 & ++++ & ++++ & +++ & +++ & - & ++ & ++ & +++ & ++ & ++ & - & ++++ & +++ \\
\hline 09 & KC Zuzana C2 & ++++ & ++++ & +++ & + & - & - & ++++ & ++++ & + & + & - & ++++ & +++ \\
\hline 10 & Kompolti & ++++ & ++++ & +++ & ++ & - & ++ & + & ++ & - & - & - & +++ & ++ \\
\hline 11 & Monoica & ++++ & ++++ & ++ & ++++ & + & ++++ & +++ & ++++ & + & ++ & +++ & +++ & ++ \\
\hline 12 & Silvana & ++++ & ++++ & +++ & +++ & - & + & ++ & ++++ & ++ & + & - & +++ & +++ \\
\hline 13 & Tiborszallasi & ++++ & ++++ & +++ & ++ & - & + & +++ & ++++ & + & + & - & ++++ & +++ \\
\hline 14 & Tisza & ++++ & ++++ & ++++ & +++ & - & + & +++ & +++ & ++ & + & - & ++++ & +++ \\
\hline 15 & Uso 31 & ++++ & ++++ & +++ & +++ & + & ++ & +++ & +++ & ++ & ++ & - & +++ & +++ \\
\hline 16 & Zenit & ++++ & +++ & +++ & ++ & - & + & +++ & ++++ & +++ & +++ & - & +++ & +++ \\
\hline \multicolumn{15}{|c|}{ drug-type } \\
\hline 17 & Afghani \#1 & - & ++++ & - & ++++ & + & ++++ & - & - & - & +++ & +++ & +++ & +++ \\
\hline 18 & Black Domina & - & ++++ & - & ++++ & ++ & ++++ & +++ & ++++ & ++ & ++ & +++ & +++ & +++ \\
\hline 19 & Durban & - & ++++ & - & ++++ & - & ++++ & - & ++++ & - & + & + & ++ & ++ \\
\hline 20 & Jack Flash \#5 & - & ++++ & - & ++++ & ++ & ++++ & +++ & ++++ & ++ & +++ & ++++ & ++++ & +++ \\
\hline 21 & Jack Herer & - & ++++ & - & ++++ & + & ++++ & - & - & - & ++ & +++ & +++ & +++ \\
\hline 22 & Jamaican Pearl & - & ++++ & - & ++++ & ++ & ++++ & +++ & +++ & + & +++ & +++ & +++ & +++ \\
\hline 23 & Northern Lights & - & ++++ & - & ++++ & + & ++++ & +++ & ++++ & + & + & +++ & +++ & +++ \\
\hline 24 & Northern Lights \#5 x Haze & - & ++++ & - & ++++ & ++ & ++++ & +++ & +++ & ++ & +++ & +++ & +++ & ++++ \\
\hline 25 & Sensi Skunk & - & ++++ & - & ++++ & + & ++++ & - & - & - & + & ++ & +++ & +++ \\
\hline 26 & Shiva Skunk & - & ++++ & - & ++++ & + & ++++ & - & - & - & ++ & +++ & +++ & +++ \\
\hline 27 & Silver Haze & - & ++++ & - & ++++ & + & ++++ & +++ & ++++ & ++ & +++ & ++++ & +++ & ++ \\
\hline
\end{tabular}

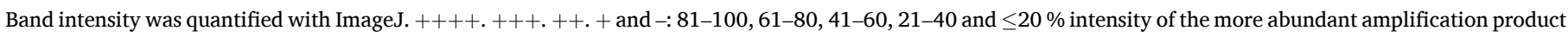
obtained with the 13 primer pairs, respectively.

THCAS and CBDAS sequences represent only a minimal part of hemp germplasm, thus the possibility exists that with cultivars not included in the above sequence analysis the designed primer pairs would not result into the expected amplification pattern. To investigate this aspect, DNA was extracted from single seeds of a set of 16 fiber-type and 11 drug-type varieties (Table 2). Among them, for only 3 (namely Carmagnola selezionata, Futura 75 and Uso 31) and 1 (Northern Lights) THCAS and CBDAS sequences were available, respectively [22]. PCR was carried out with the 12 putatively specific primer pairs, plus a thirteenth pair (T) designed to amplify a region that had been found to be highly conserved in both THCAS and CBDAS (Supplementary Fig. S3T), to be used as a positive control. DNA was not quantified, and low stringency conditions and high number of cycles were used to ensure amplification. Three different seeds for each genotype were separately extracted and analysed, and consistent results were obtained without exceptions. Results, summarized in Table 2, pointed out a heterogeneous picture. With primer pair $\mathrm{T}$, despite conservation in all known sequences, amplification was not obtained in one case (Fedora 17). Considering the other, putatively specific primer pairs, in most cases results differed significantly from the expected amplification patterns. For instance, with primer pair L, designed to amplify THCAS from drug-type varieties, amplification was obtained also with fifteen out of sixteen fiber-type varieties. With pairs $\mathrm{D}$ and $\mathrm{F}$, designed to amplify CBDAS from drug-type varieties, amplification occurred also with several cultivars of the other chemotype. This notwithstanding, consistent results were obtained with primer pair A, designed to amplify CBDAS from fiber-type varieties, and pair $\mathrm{K}$, designed to amplify THCAS from drug-type varieties. In both theses cases all target sequences were identified, while non-target sequences did not result in amplicon formation, with only 2 exceptions out of 16 for primer pair K (Supplementary Fig. S5).

The possibility of using these two primer pairs for molecular discrimination of hemp from cannabis was therefore investigated further on three new preparations, for which DNA was purified and quantified. When decreasing quantities of template were used, amplification in target samples was obtained with DNA amounts as low as $1 \mathrm{ng}$ (Fig. 2A), whereas no amplicons of the expected size were evident with 100-fold higher amounts of DNA from tobacco or other plants species (Supplementary Fig. S6). By increasing the annealing temperature and using a fixed amount of template (10 ng), optimal results were obtained at $55{ }^{\circ} \mathrm{C}$, confirming previous data for primer pair A (Fig. 2B). At this stringency, amplification in non-target DNAs with primer pair $\mathrm{K}$ was reduced to a single case (Fig. 2C).

\section{Discussion}

The availability of molecular markers to distinguish legal from illegal hemp varieties would represent a very attractive result, greatly facilitating the resurgence of this crop as an agricultural commodity worldwide. The attainment of this goal was initially hampered by the erroneous assumption that THCAS and CBDAS were allelic and codominant [17-21]. A recent study in which CBDAS and THCAS from 11 drug-type and 10 fiber-type hemp varieties were on the contrary sequenced in parallel, reported the identification of some SNPs that were hypothesized as signatures for decreased THCAS activity in fiber-type plants, and deletions possibly resulting in CBDAS loss-of-function in drug-type plants [22]. Nevertheless, the presence of these possible markers was investigated only in those 21 varieties, and the actual ability of the corresponding primers to discriminate between chemotypes was not assessed experimentally. The rationale of the present work was, therefore, to re-evaluate all the information previously made available under a correct perspective, where CBDAS and THCAS are two distinct genes present in both drug-type and fiber-type plants.

A wide set of 38 CBDAS and 145 THCAS sequences, for 36 and 67 of which the chemotype was known, was considered. A Neighbour-joining tree generated from the aligned sequences not only confirmed the divergence between the two genes, suggesting that accession AB292683.1 should be re-annotated as THCAS, but clearly showed the presence within each cluster of two subgroups in which almost all 

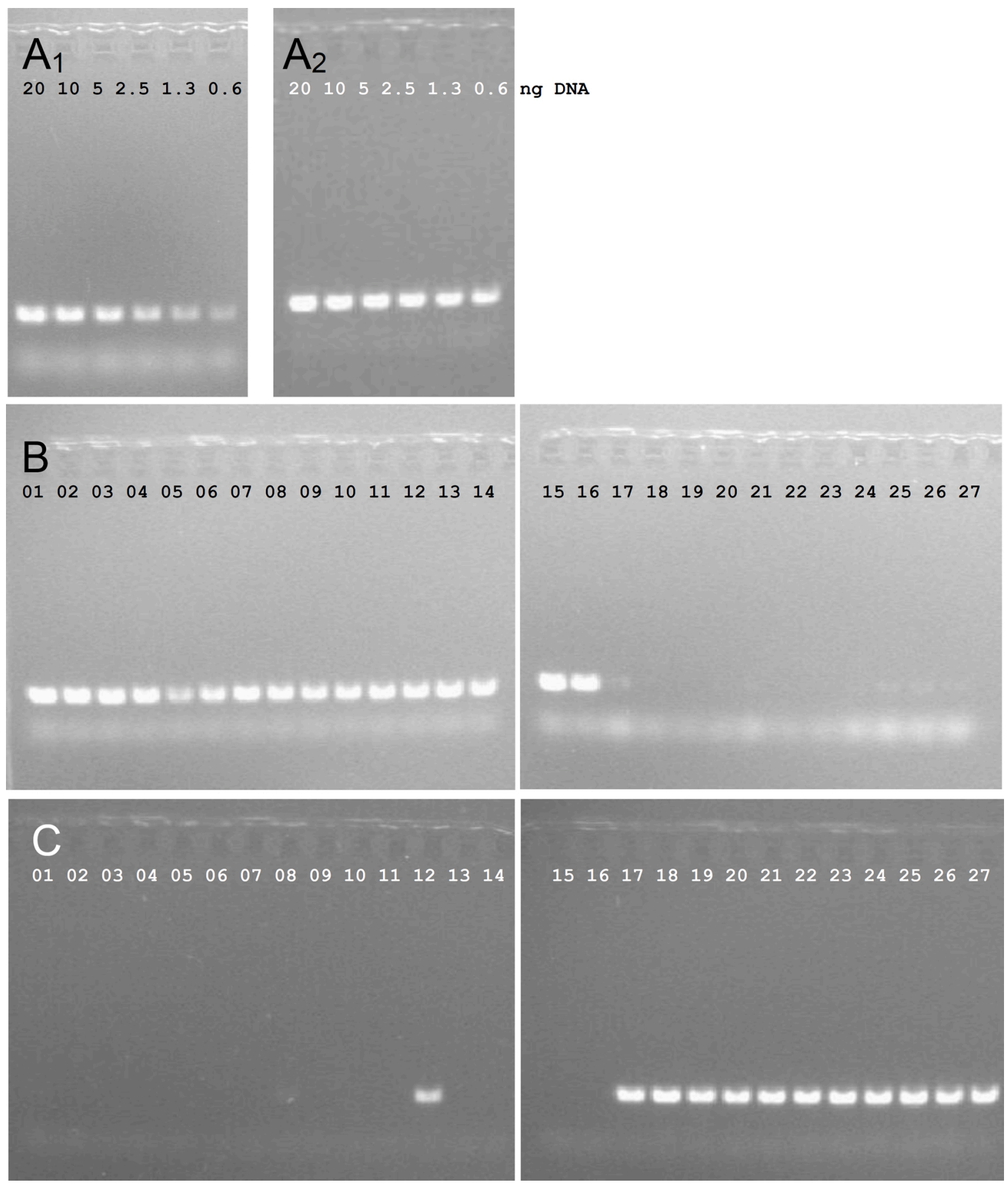

Fig. 2. The patterns of DNA amplicons obtained with primer pairs A (Panel B; exposure time $0.28 \mathrm{~s}$ ) and $\mathrm{K}$ (Panel C; exposure time $0.12 \mathrm{~s}$ ) using $10 \mathrm{ng}$ template DNA from fiber-type (01-16, as listed in Table 2) or drug-type (17-27) hemp varieties. In panel A, decreasing amounts of template DNA from Carmagnola selezionata ( $\left.\mathrm{A}_{1}\right)$ or Afghani \#1 $\left(\mathrm{A}_{2}\right)$ were amplified with primer pairs A and $\mathrm{K}$, respectively. In all cases annealing was carried out at $55^{\circ} \mathrm{C}$.

sequences of a given chemotype co-clustered. In the case of $C B D A S$, for which a lower number of sequences were available, the two clades perfectly resolved drug-type from fiber-type varieties. For THCAS, with a double number of sequences available, the two clades were well separated, but four accessions clustered in the erroneous subgroup. This inconsistency may depend on a wrong classification of their chemotype, or on the presence of drug-type alleles in fiber-type varieties. Indeed, recent results suggested that synthases for the cannabinoid pathway are highly duplicated [28]. Whatever the reasons for these few exceptions, the whole picture strongly suggested that in most cases drug-type hemp varieties contain both THCAS and CBDAS forms that are dissimilar from those in fiber-type cultivars, and that these differences could be used to identify SNPs able to discriminate the chemotypes.

With this aim, three primer pairs were identified for each subgroup whose complementary sequences are present in virtually all target varieties, while showing a significant number of SNPs in most sequences belonging to the other three, non-target subgroups. Other putative signatures were disclosed within the two genes, but at this stage of the research only the 12 primer pairs described in Table 1 were considered. When these primers were used to analyse DNA extracted from a number of mostly unsequenced hemp varieties, the patterns obtained were in general strikingly different from the expected ones. Such inconsistent results may depend in part on the fact that no attempts were initially made to optimize PCR conditions. We aimed at assessing the potentiality 
of a very simple protocol in which DNA is extracted from a specimen as small as a single seed, and an end-point PCR is performed even without template DNA quantification. Also the amount of the amplification products was roughly estimated by image analysis following gel visualization.

This notwithstanding, these preliminary results permit drawing some conclusions. A few genetic signatures in CBDAS and THCAS sequences are indeed present that may contribute to distinguish hemp chemotypes. Despite the basic protocol adopted, in the case of primer pairs $\mathrm{A}, \mathrm{C}$ and $\mathrm{K}$, consistent results (i.e. amplification in target genotypes and lack of amplification in non-target genotypes) were obtained in 27, 26 and 25 out of 27 varieties tested, respectively (Supplementary Fig. S5). For the two most promising primer pairs, A and K, the analysis was repeated with purified DNA preparations. An increase of stringency with the adoption of higher annealing temperatures and the normalization of the concentration of the template in the reaction mixture allowed to improve discrimination, and overcome some of the inconsistencies found (Fig. 2C).

On the other hand, data confirmed previous studies showing an extreme genetic variability in hemp germplasm concerning these genes. Coupled with the possible occurrence of multiple gene copy numbers [28] leading to the presence of drug alleles in fiber varieties and vice-versa, this implies that the identification of a single SNP able in all cases to discriminate the two chemotypes is unlikely. Therefore, other similarly informative SNPs should be further identified, and used together to genotype a large number of hemp varieties. The availability of a well-characterized SNPs panel could lead to a simplified and standardized analysis with fluorescent primers in a multiplex. In such a way, the building of a detailed database showing the corresponding amplification patterns in as many hemp cultivars as possible would be useful not only for chemotype DNA barcoding, but also for varietal identification, another essential application for breeding programs and seed patent protection. Work is currently in progress with this aim.

\section{Acknowledgements}

The authors thank Dr Andrea Guandalini for help in primer design, and Prof Chiara Scapoli for critical reading of the manuscript. This work was supported in part by a grant from the University of Ferrara (FAR 2018).

\section{Appendix A. Supplementary data}

Supplementary material related to this article can be found, in the online version, at doi:https://doi.org/10.1016/j.fsigen.2021.102464.

\section{References}

[1] C. Schluttenhofer, L. Yuan, Challenges towards revitalizing hemp: a multifaceted crop, Trends Plant Sci. 22 (2017) 917-929.

[2] J. Fike, Industrial hemp: renewed opportunities for an ancient crop, Crit. Rev. Plant Sci. 35 (2016) 406-424.

[3] M. Tyler, J. Shepherd, D. Olson, W. Snell, S. Proper, S. Thornsbury, Economic Viability of Industrial Hemp in the United States: a Review of State Pilot Programs, EIB-217, U.S. Department of Agriculture, Economic Research Service, 2020.

[4] I. Adesina, A. Bhowmik, H. Sharma, A. Shahbazi, A review on the current state of knowledge of growing conditions, agronomic soil health practices and utilities of hemp in the United States, Agriculture 10 (2020) 129.
[5] R. Abuhasira, L. Shbiro, Y. Landschaft, Medical use of cannabis and cannabinoids containing products - regulations in Europe and North America, Eur. J. Intern. Med. 49 (2018) 2-6.

[6] D.W. Lachenmeier, S.G. Walch, Analysis and toxicological evaluation of Cannabinoids in hemp food products-a review, Electr. J. Environ. Agric. Food Chem. 4 (2005) 812-826.

[7] M. Hädener, S. König, W. Weinmann, Quantitative determination of CBD and THC and their acid precursors in confiscated cannabis samples by HPLC-DAD, Forensic Sci. Int. 299 (2019) 142-150.

[8] T.J. Raharjo, I. Widjaja, S. Roytrakul, R. Verpoorte, Comparative proteomics of Cannabis sativa plant tissues, J. Biomol. Tech. 15 (2004) 97-106.

[9] M.D. Marks, L. Tian, J.P. Wenger, S.N. Omburo, W. Soto-Fuentes, J. He, D.R. Gang, G.D. Weiblen, R.A. Dixon, Identification of candidate genes affecting $\delta^{9}$. tetrahydrocannabinol biosynthesis in Cannabis sativa, J. Exp. Bot. 60 (2009) 3715-3726.

[10] E.E. Hakki, S.A. Kayis, E. Pinarkara, A. Sag, Inter simple sequence repeats separate efficiently hemp from marijuana (Cannabis sativa L.), Electron. J. Biotechnol. 10 (2007) 570-581.

[11] G. Piluzza, G. Delogu, A. Cabras, S. Marceddu, S. Bullitta, Differentiation between fiber and drug types of hemp (Cannabis sativa L.) from a collection of wild and domesticated accessions, Genet. Resour. Crop Evol. 60 (2013) 2331-2342.

[12] M. Kojoma, H. Seki, S. Yoshida, T. Muranaka, DNA polymorphisms in the tetrahydro-cannabinolic acid (THCA) synthase gene in "drug-type" and "fiber-type" Cannabis sativa L, Forensic Sci. Int. 159 (2006) 132-140.

[13] S. Sirikantaramas, S. Morimoto, Y. Shoyama, Y. Ishikawa, Y. Wada, Y. Shoyama, F. Taura, The gene controlling marijuana psychoactivity: molecular cloning and heterologous expression of $\delta^{1}$-tetrahydrocannabinolic acid synthase from Cannabis sativa L, J. Biol. Chem. 279 (2004) 39767-39774.

[14] M.A. El Alaoui, M. Melloul, S.M. Udupa, E.E. Hakki, H. Stambouli, A. El Bouri, S. A. Amine, A. Soulaymani, E. El Fahime, Study of Moroccan Cannabis sativa DNA polymorphism in the THCA synthase gene from seized Moroccan cannabis resin (Hashish), J. Plant Biol. Res. 5 (2016) 1-11.

[15] D. Rotherham, S.A. Harbison, Differentiation of drug and non-drug Cannabis using a single nucleotide polymorphism (SNP) assay, Forensic Sci. Int. 207 (2011) 193-197.

[16] K.U. Laverty, J.M. Stout, M.J. Sullivan, H. Shah, N. Gill, L. Holbrook, G. Deikus, R. Sebra, T.R. Hughes, J.E. Page, H. van Bakel, A physical and genetic map of Cannabis sativa identifies extensive rearrangements at the THC/CBD acid synthase loci, Genome Res. 29 (2019) 146-156.

[17] F. Taura, S. Sirikantaramas, Y. Shoyama, K. Yoshikai, Y. Shoyama, S. Morimoto, Cannabidiolic-acid synthase, the chemotype-determining enzyme in the fiber-type Cannabis sativa, FEBS Lett. 581 (2007) 2929-2934.

[18] E.P. de Meijer, M. Bagatta, A. Carboni, P. Crucitti, V.M. Moliterni, P. Ranalli, G. Mandolino, The inheritance of chemical phenotype in Cannabis sativa L, Genetics 163 (2003) 335-346.

[19] C. Staginnus, S. Zörntlein, E. de Meijer, A PCR marker linked to a THCA synthase polymorphism is a reliable tool to discriminate potentially THC-rich plants of Cannabis sativa L, J. Forensic Sci. 59 (2014) 919-926.

[20] C. Onofri, E.P.M. de Meijer, G. Mandolino, Sequence heterogeneity of cannabidiolic- and tetrahydrocannabinolic acid-synthase in Cannabis sativa L. and its relationship with chemical phenotype, Phytochemistry 116 (2015) 57-68.

[21] G.D. Weiblen, J.P. Wenger, K.J. Craft, M.A. El Sohly, Z. Mehmedic, E.L. Treiber, M. D. Marks, Gene duplication and divergence affecting drug content in Cannabis sativa, New Phytol. 208 (2015) 1241-1250.

[22] F. Cascini, A. Farcomeni, D. Migliorini, L. Baldassarri, I. Boschi, S. Martello, S. Amaducci, L. Lucini, J. Bernardi, Highly predictive genetic markers distinguish drug-type from fiber-type Cannabis sativa L, Plants (Basel) 8 (2019) 496.

[23] K.J. Mc Kernan, Y. Helbert, V. Tadigotla, S. Mc Laughlin, J. Spangler, L. Zhang, D. Smith, Single molecule sequencing of THCA synthase reveals copy number variation in modern drug-type Cannabis sativa L, bioRxiv (2015), https://doi.org/ 10.1101/028654.

[24] F. Madeira, Y.M. Park, J. Lee, N. Buso, T. Gur, N. Madhusoodanan, P. Basutkar, A. R.N. Tivey, S.C. Potter, R.D. Finn, R. Lopez, The EMBL-EBI search and sequence analysis tools APIs in 2019, Nucleic Acids Res. 47 (2019) W636-W641.

[25] A. Boc, Diallo, B. Alpha, V. Makarenkov, T-REX: a web server for inferring, validating and visualizing phylogenetic trees and networks, Nucl. Acids Res. 40 (2012) W573-W579.

[26] Y.T. Huang, J.I. Yang, M. Chrobak, J. Borneman, PRISE2: software for designing sequence-selective PCR primers and probes, BMC Bioinf. 15 (2014) 317.

[27] C.A. Schneider, W.S. Rasband, K.W. Eliceiri, NIH Image to ImageJ: 25 years of image analysis, Nat. Methods 9 (2012) 671-675.

[28] D. Vergara, E.L. Huscher, K.G. Keepers, R.M. Givens, C.G. Cizek, A. Torres, R. Gaudino, N.C. Kane, Gene copy number is associated with phytochemistry in Cannabis sativa, AoB Plants 11 (2019) plz074. 